\title{
Factitious Hemoperfusion Based ROI Optimization in PPGi Analysis and its Application for Blood Pressure Determination
}

\author{
Fubo Deng ${ }^{1}$ Xiaoshan $\mathrm{Zhu}^{2^{*}}$ and Yunfang Jia ${ }^{1 *}$ \\ ${ }^{1}$ College of Electronic Information and Optical Engineering, Nankai University, China
}

${ }^{2}$ Department of Electrical and Biomedical Engineering, University of Nevada Reno, USA

Received: 眥 January 10, 2019; Published: 眥 January 16, 2019

*Corresponding author: Yunfang Jia, College of Electronic Information and Optical Engineering, Nankai University, China 300071

Xiaoshan Zhu, Department of Electrical and Biomedical Engineering, University of Nevada Reno, USA 89557

\begin{abstract}
Photoplethysmography Imaging (PPGi) is an emerging technology to monitor physiological parameters. However, the signal-to-noise ratio in PPGi usually is low due to inappropriate selection of Region of Interest (ROI). Herein, to enhance the signal-to-noise ratio, an optimization approach for ROI selection is proposed based on factitious hemoperfusion. Factitious hemoperfusion is able to amplify instantaneous blood volume and augment the volume of the blood vessel, and such an amplification will facilitate the identification of dense vessel regions as the optimized ROIs for PPGi. Furthermore, a self-adaptive PPGi denoising method is created to purify PPGi data, and an approach calculating Blood Pressure (BP) from PPGi signals is proposed and developed. Through comparing the calculated BP values to the ones measured using a commercial sphygmomanometer, it is found that the BP values resulted from the optimized ROIs are closer to the measured ones (with an error rate less than 5\%).
\end{abstract}

Keywords: Photoplethysmography Imaging; Region of Interest; Blood Pressure; Windkessel Model

Abbreviations: PET: Photo-Electronic Transformation; BP: Blood Pressure; PPGi: Photoplethysmography Imaging; PPG: Photoplethysmography; SNR: Signal-to-Noise Ratio; ROI: Region of Interest; BP-PPGi: BP from PPGi; BP-CSM: BP by the Commercial Sphygmomanometer; HP-PPGi: Estimate the High Pressure; LP-PPGi: Estimate the Low Pressure; HP-CSM: High Pressure which are Measured by CSM; LP-CSM: Low Pressure which are Measured by CSM

\section{Introduction}

The heart rhythms accompanied by systole and diastole can change the blood volume in the vessels under the skin surface, and further alter the light absorption/reflection of vessels. Thus, the changes on the light absorption/reflection of vessels can be detected by a photoelectronic component. Based on this PhotoElectronic Transformation (PET) principle [1] technologies for monitoring physiological parameters (like heart rate [2], Blood Pressure (BP) [3], respiratory rate [4] etc.,) have been developed. Among these technologies, Photoplethysmography Imaging (PPGi) is an emerging trend. PPGi is an evolved photoplethysmography (PPG) [5] with the merits of noncontact and remote sensing [6]. Particularly, for patients with skin injuries, refusing treatments or demanding long-term supervision, PPGi is an ideal preference to monitor their physiological parameters.

To acquire correct vital signs, three parts are needed in the PPGi system, which include video collection, image processing and algorithm to extract physiological information. There have been lots of reported works for each of them. For instance, studies about video collection are mainly focused on camera [7], light-source [8] and their spatial distribution [9]; nowadays, phone collected [10] and ambient light excited [11] PPGi signal collections have been realized. Image processing calculation is an inescapable step in PPGi technique, in order to get a correct and valuable waveform, lots of strategies have been exploited to enhance the Signal-to-Noise Ratio (SNR), such as the methods of motion compensation [12,13], Fourier Transform (FFT) [14], wavelet transform [15,16]. To facilitate PPGi's applications, there are efforts in extracting physiological parameters from PPGi data. For examples, the value of relative oxygen saturation can be estimated by mapping multi-layered blood perfusion and structuring opto-physiological model [17]; the breathing frequency is tracked via differential signal processing algorithm with the maximized signals and the minimized noise [18]; based on head motions the pulse rate variability is extracted from blood volume pulse by fusing partial color channel signals of multiple imagers, source signal separation and the autoregressive model [19], etc. In conclusion, it has been demonstrated that PPGi is a potential and reliable vital sign monitoring technique [20]. 
Nevertheless, PPGi is not as popular as PPG, because of its unsatisfactory waveform, stability and SNR which are inherent in its remote video collection. Although the SNR of PPGi can be improved by optimizing hardware configuration such as light source, camera, background, and so on $[8,14,17]$ appropriate approaches to optimize the selection of the Region of Interest (ROI) and therefore enhance the PPGi SNR have not be explored yet. In this work, a scheme to optimize ROI selection is firstly proposed based on factitious hemoperfusion. The proposed ROI optimizing method is examined and analyzed through the comparisons of the waveform and SNR of the PPGi signals which are collected under the in-respective lights of red, green, blue and white. Afterwards, a
SNR self-adaptive PPGi denoising method is developed based on the wavelet transform, Butterworth low-pass filter and median filter, the collected PPGi signals from the optimized ROI are pretreated by this method. The de-noised PPGi is ready for the following BP estimation. Lastly, the Windkessel model, a classical mathematical model of the cardiovascular system, is exploited to excavate physiology information (BP) from the de-noised PPGi signal, for the first time, to the best of our knowledge. Our proposed strategy of achieving BP from PPGi (BP-PPGi) is confirmed by evaluating the error rates of BP-PPGi with the measured ones by a commercial sphygmomanometer (BP-CSM). It is found that the best error rate $(<5 \%)$ is from the PPGi data at optimized ROI.

\section{Materials and Method}

\section{PPGi System}
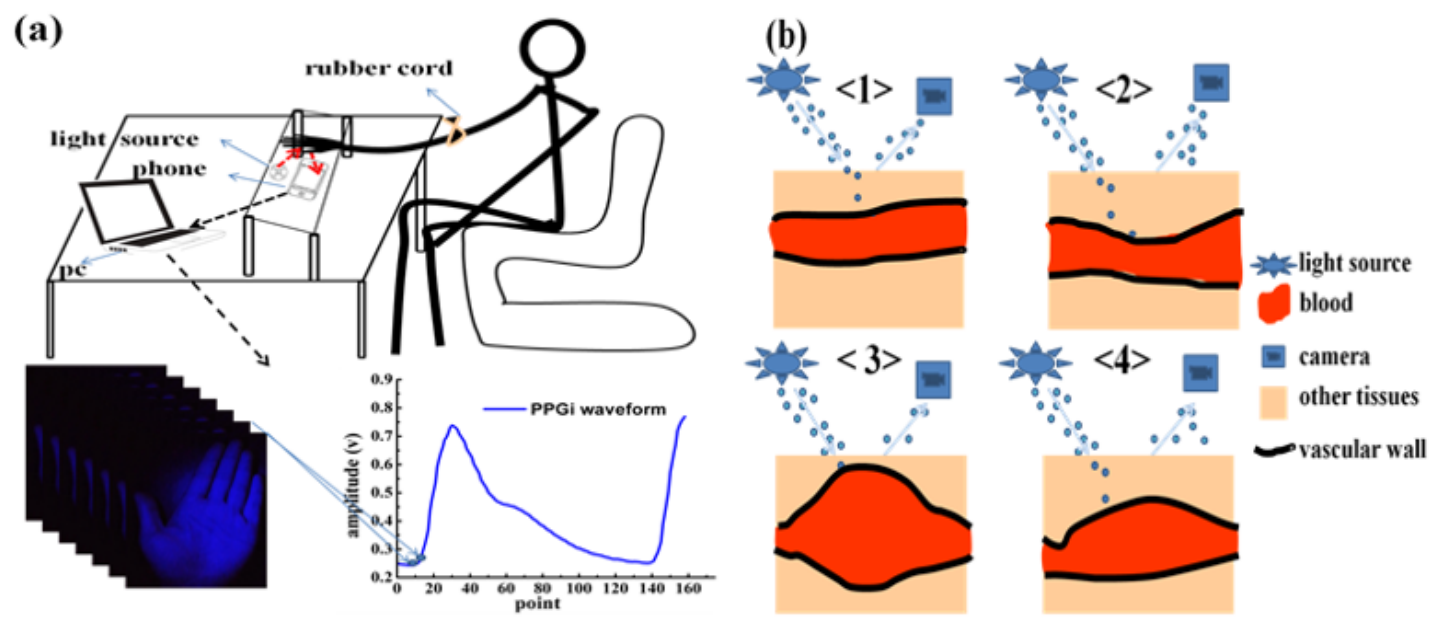

Figure 1: Diagrams for the measuring setup (a) and ROI selection principle (b) of PPGi technology. In (a), the experimental setup includes the light source, phone and computer. The phone is used to capture and store the of-interest PPGi data. The light source is used to excite the desired irradiation, four kinds of light are examined in this work which are red, green, blue and white. The computer is used to execute the proposed algorithms to extract PPGi images and waveforms, as shown in (a). Moreover, the factitious hemoperfusion used for the ROI selection is illustrated by the rubber cord being tied around the elbow. In (b), there are four states of blood volume in vessel which are: $<1>$ normal state, $<2>$ the vessel is tightened and blood volume almost constant, $<3>$ the tightened vessel is loosened and blood is poured into the vessel, $<4>$ the vessel and blood in it are recovered to its normal state.

Our PPGi system adopting factitious hemoperfusion is illustrated in Figure 1a. The altered blood volume in the vessels caused by factitious hemoperfusion is illustrated in Figure $1 \mathrm{~b}$. There are three parts in this system, which are the illumination unit to generate the light beam, the photodetector (like camera, mobile phone, etc.) to detect the reflected light, and the computer to execute our proposed calculations. LEDs are used for illumination, and their colors are red $(720 \mathrm{~nm})$, green $(530 \mathrm{~nm})$, blue $(480 \mathrm{~nm})$ and white, respectively. They have tunable power from $0.5 \mathrm{~W}$ to 1 $\mathrm{W}$, and their radiating range can be up to $20 \mathrm{~cm} \times 20 \mathrm{~cm}$. A mobile smart phone (HONOR 9, HUAWEI, China) with $1920 \times 1080$ pixels and 30 frame s- 1 is used as the photodetector, and the PPGi data can also be collected and stored by this phone. The collected data are analyzed by MATLAB R2014b 8.4.0. The geometry of the PPGi detection system is depicted in Figure 1a, the target object (some area of human body) is placed on the upper side of the system, the LEDs and the mobile smart phone are located at the same horizontal plane with the distance of about $4 \mathrm{~cm}$. The distance between the target object and the plane coexisting with the LEDs and mobile phone is about $15 \mathrm{~cm}$. The measuring time is around 30 seconds. Simultaneously, a parallel detection is executed to get BP-CSM using HEM7051 (OMRON, Japan), which functions as a control.

\section{Decision of ROI}

The decision for ROI selection is very important, since the blood volume and distribution in different body parts are distinctive, because of the deviations in the accumulating states of arteries, veins, and capillaries [21]. In the blood vessel intensive regions, the quantity of blood volume can be varied more obviously than that of other regions, then a stronger PPGi signal can be captured. The most commonly used ROIs are hand and forehead, because of their operational convenience and the vessels in them are dense and close to the heart. In this work, more efforts are made to optimize ROI from forehead and hand. Our approach for the ROI selection is described as follows. 
Diagrams for the measuring setup (a) and ROI selection principle (b) of PPGi technology. In (a), the experimental setup includes the light source, phone and computer. The phone is used to capture and store the of-interest PPGi data. The light source is used to excite the desired irradiation, four kinds of light are examined in this work which are red, green, blue and white. The computer is used to execute the proposed algorithms to extract PPGi images and waveforms, as shown in (a). Moreover, the factitious hemoperfusion used for the ROI selection is illustrated by the rubber cord being tied around the elbow. In (b), there are four states of blood volume in vessel which are: $<1>$ normal state, $<2>$ the vessel is tightened and blood volume almost constant, $<3>$ the tightened vessel is loosened and blood is poured into the vessel, $<4>$ the vessel and blood in it are recovered to its normal state.

Firstly, PPGi video is acquired from the forehead and the entire hand, through comparisons of their waveforms and SNR, then a better body part for PPGi analysis is confirmed. Secondly, to enhance the PPGi analyzing efficiency, accuracy and speed, we designed an interesting experiment for selecting a more optimized small-range ROI, by the method of amplifying instantaneous blood volume, named as factitious hemoperfusion here. As shown in Figure 1a, the factitious hemoperfusion is realized through tying a rubber cord around the elbow, for 10 to 15 seconds, and then loosening it. During the period of factitious hemoperfusion, the PPGi signals are collected. It is deduced there should be changed blood volume in the vessel of the tied body, as shown in Figure 1b [22]. In the state $<1>$ which corresponds to the moment before factitious hemoperfusion, the blood vessel is normal state and PPGi signal can be got. In the state $<2>$, the blood vessel is tightened, and the blood volume in them is very small. At this state, the light absorbance in this region is lowered, and the slightly disturbed waveform of PPGi is detected. In the state $<3>$, at the moment of loosening the rubber cord, accompanied by the cardiac rhythm, a large amount of blood (more than the normal condition) is poured into the hand from the heart. With the increasing of blood volume in hand, the external light absorption can be increased greatly, and then reflected light collected by the camera is greatly reduced. Thus, the amplitude of the PPGi waveform is rapidly reduced, and ultimately restored to the original level (as shown in Figure $1 \mathrm{~b}<4>$ ). All in all, the changing of blood volume in Figure $1 \mathrm{~b}$ will be different depending on vessel density, then the varied PPGi amplitude can be measured according to the principle of PPGi. So, we can look for the blood vessel dense regions through distinguishing the changed PPGi amplitudes, then the optimized ROI can be decided.

\section{Self-Adaptive PPGi Denoising for SNR}

The procedure in the proposed PPGi denoising method is outlined here: (1) original PPGi video data (in the format of .mp4) are read-in frame-by-frame as image data; (2) one-dimensional arrays of PPGi data are obtained by averaging pixels value of these image data, as shown by the part of video images and PPGi waveform of Figure1a; (3) the SNR of the data from (2) are calculated, and the calculation formula is as follows:

$$
\mathrm{SNR}=10 \log _{10} \frac{\mathrm{ps}}{\mathrm{pn}} \quad------- \text { Equation (1) }
$$

Among them, ps is signal power and pn is noise power; (4) by using the wavelet transform [23], burrs of data from (2) are removed; (5) if the value of SNR lower than the threshold value (20 at here), the Butterworth low-pass filtering [24] is used to filter high frequency noise (like odd-shaped noise peaks); on the contrary, if the SNR of (2) is more than 20, that means the high frequency noise is very small and this step can be omitted; (6) the median filter [25] is applied to eliminate an offset phenomenon and to make the waveforms smooth.

\section{Blood Pressure Estimation}

The BP estimation is conducted according to the Windkessel model which is a mathematical model established for the cardiovascular system. In this model, the arterial vascular circulatory system is compared to an equivalent circuit network. The systole of the heart is regarded as the input of the circuit, the $\mathrm{BP}$ is regarded as the voltage $\mathrm{P}$, blood flow in blood vessel is seen as current I, the resistance of the blood vessel wall and the viscosity of the blood are taken as the resistance R, the capacity of the blood vessel is seen as the capacitance $C$. The current $B P$ value is obtained based on fixed parameters $\mathrm{R}$ and $\mathrm{C}$ and the analyzed pulse wave data [26].

According to the Kirchhoff's law, the mathematical model of the equivalent circuit is expressed as follows:[27]

$$
\frac{\mathrm{P}(\mathrm{t})}{\mathrm{R}}+\mathrm{C} \frac{\mathrm{dP}(\mathrm{t})}{\mathrm{dt}}=\mathrm{I}(\mathrm{t}) \text {------ Equation (2) }
$$

The values of the parameters $\mathrm{R}$ and $\mathrm{C}$ in equation 2 are directly related to the quality of the model. Based on the least square method, their values are fitted by the standard data from the University of Queensland Vital Signs Dataset [28].

Among them, the current I can be obtained according to equation 3 , where the rising time $\mathrm{T}_{\mathrm{s}}$ is the time from $10 \%$ of rising waveform amplitude to wave peak, the falling time $T_{d}$ is the time from wave peak to $10 \%$ of falling waveform amplitude and $\mathrm{T}_{\mathrm{c}}=\mathrm{T}_{\mathrm{s}}+$ $\mathrm{T}_{\mathrm{d}}:$ [27]

$$
I=\frac{C T_{c}}{60 \int_{0}^{T_{s}} \sin \left(\pi t / T_{s}\right) d t}
$$

Combining equations 2,3, the mathematical expressions of the high BP and the low BP can be obtained, respectively: [27]

$$
\begin{gathered}
P_{s}=P_{t s} e^{T_{s} / R C}+\frac{l T_{s} C \prod R^{2}}{T_{s}^{2}+C^{2} \prod^{2} R^{2}}\left(1+e^{T_{s} / R C}\right) \text {-------- Equation (4) } \\
P_{d}=P_{t d} e^{T_{d} / R C}
\end{gathered}
$$

In these equations, $\mathrm{P}_{\mathrm{ts}}(100-120 \mathrm{mmHg})$ is the initial high pressure, $P_{t d}\left(70-90 \mathrm{mmHg}\right.$ ) is the initial low pressure, $P_{s}$ is the estimated high pressure, and $\mathrm{P}_{\mathrm{d}}$ is the estimated low pressure. 


\section{Results}

In this proof-of-concept study, eight volunteers were randomly selected (two men and six women, between twenty and thirtyfive years old) and participated in the experiments, and many measurements were made for each of them. During PPGi detecting periods, they are asked to sit quietly and have a nature breath. Meanwhile, the parameters $\mathrm{R}$ and $\mathrm{C}$ are fitted by the standard data from the University of Queensland Vital Signs Dataset.

\section{Selection of ROI}
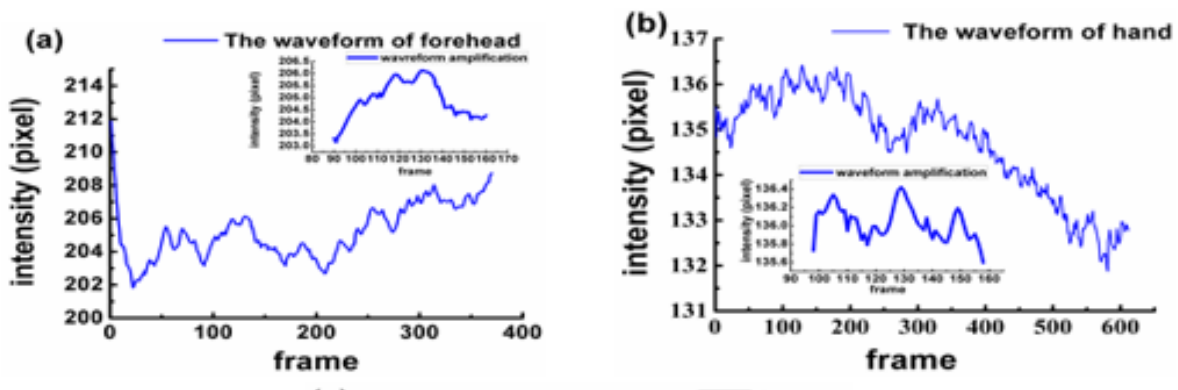

(c)
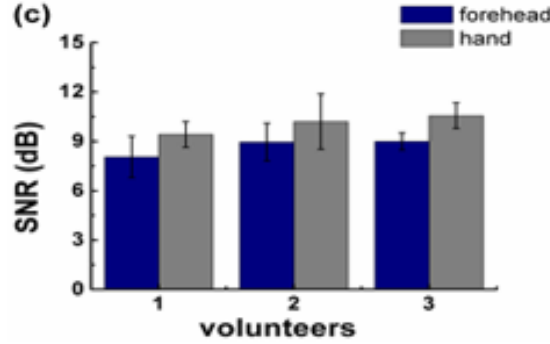

Figure 2: Results for the waveforms of forehead (a), entire hand (b) and their SNRs (c). In (a) and (b), the vertical axis is the pixel value of the image, and the horizontal axis is the number of captured image frame; the data of y-axis are calculated average values of each frame data. The experiment is executed in the same condition. In (c), the SNR values are averaged from ten times of measurements of three different volunteers which are randomly selected, and others have the similar results, and the error bars show their standard deviations.

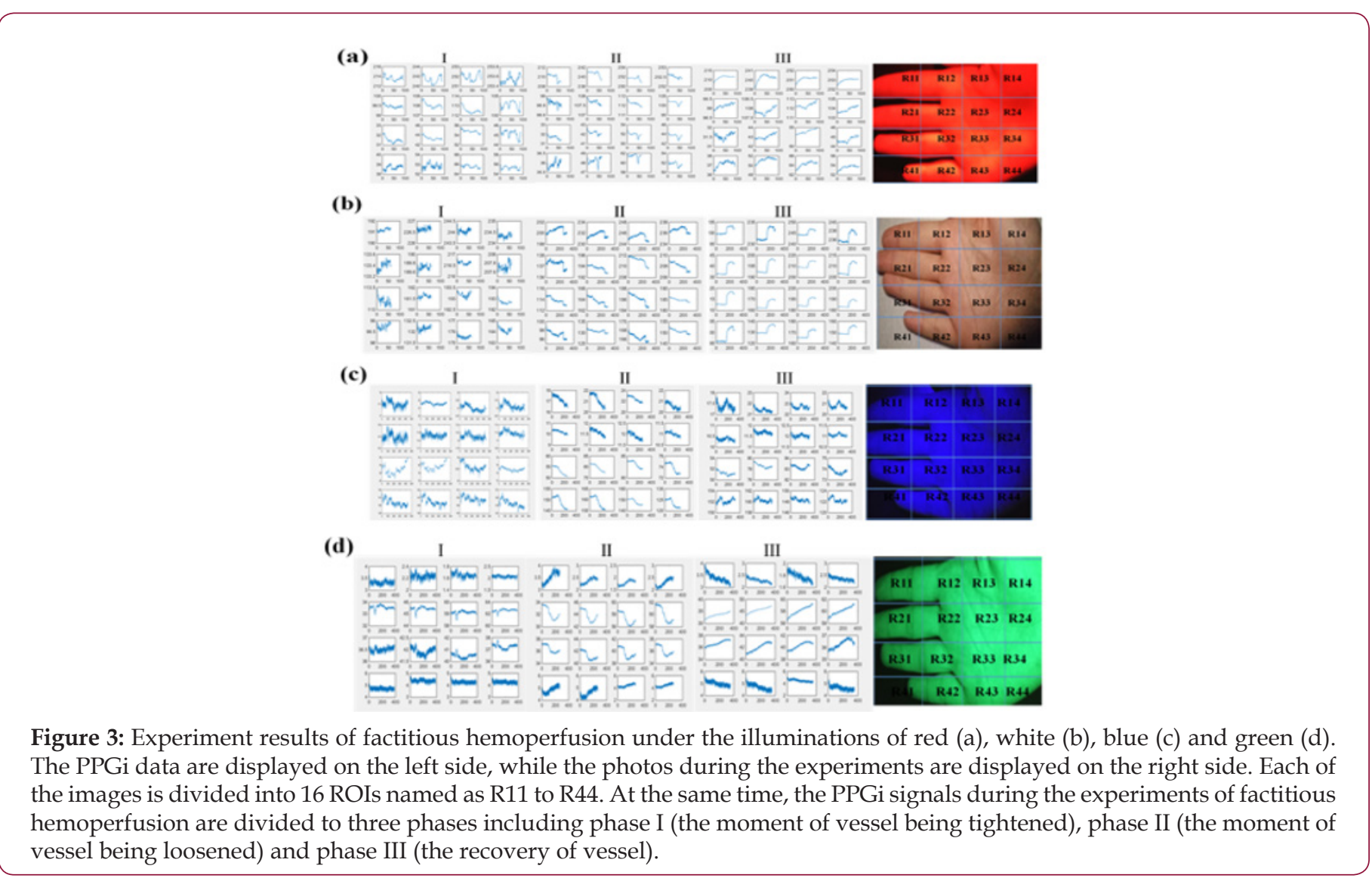

Cite this article: Fubo D, Xiaoshan Z, Yunfang J. Factitious Hemoperfusion Based ROI Optimization in PPGi Analysis and its Application for Blood Pressure Determination. Biomed J Sci \& Tech Res 13(1)-2019. BJSTR. MS.ID.002356. DOI: 10.26717/ BJSTR.2019.13.002356. 
At first, the videos of forehead and entire hand were collected by PPGi. As shown in Figures 2a and 2b, there are whole waveforms of PPGi signal from averaged pixel value of forehead and entire hand and magnified local waveform in same time range (90 to 160 frame approximately), which are shown by the insets. By comparing waveforms, the shape of the pulse wave from entire hand is more pronounced, as shown in the Figures $2 \mathrm{a}$ and $2 \mathrm{~b}$. Through the observations of the insets in Figures $2 a$ and $2 b$, it is found that the pulse wave of forehead is submerged in noise and almost invisible, and in contrast, though the waveform of entire hand has a lot of small burrs, the pulse wave contained in it can still be identified. Furthermore, the calculated SNR from hand is greater than that from forehead, as shown in Figure 2c, which suggests that the PPGi signal from hand is more meaningful. Then, more exact selection of ROI from hand is executed under different lights' illuminations according to the factitious hemoperfusion experiment mentioned in section 2.3. The video image of the entire hand is divided into 16 parts (R11 to R44) to find a small-scaled dense vessel region. Further the collected video is divided into three phases according to the experimental method mentioned in section 2.3 and illustrated in Figure 1b: (I) the curb phase, (II) the instant of blood perfusion, and (III) the recovery process. The variations of PPGi waveforms can be seen in Figure 3a. These variations include: (1) In phase I, the blood volume of vessel has no significant change, and the normal waveform can be obtained; (2) In phase II, which is equivalent to the switching from $<2>$ to $<3>$ in Figure 1 b, bloods are influxes into the vessel of hand, resulting in a greatly reduced in amplitude of waveform; (3) in phase III, the blood volume rapidly increases in the hand and the amplitude of waveform rises quickly and ends up in the normal state. It can be found that there are obvious differences among the divided parts. As shown in phase II of Figure 3a, the largest change in the instantaneous declining amplitude is obtained from R11 instead of other R0Is. The similar results are found in Figures $3 \mathrm{~b}-3 \mathrm{~d}$. Moreover, the illumination induced discrepancy is also found. For the illuminations of red, white and blue, there are consistent changing tendencies in the waveforms from different ROIs, as shown in Figures 3a-3c. However, for the green light in Figures $3 \mathrm{~d}$, the amplitude of waveforms in the first and fourth lines are rising, but in second and third lines, the waveforms are falling.

To increase the accuracy of the experimental results, the waveforms and the SNRs of R11, R23, and R24 are compared, respectively. As shown in Figures 4a-4c, the pulse wave of R11 is more obvious than that of R23 and R24, which is evidenced by the biggest SNR of R11 in Figure 4d.
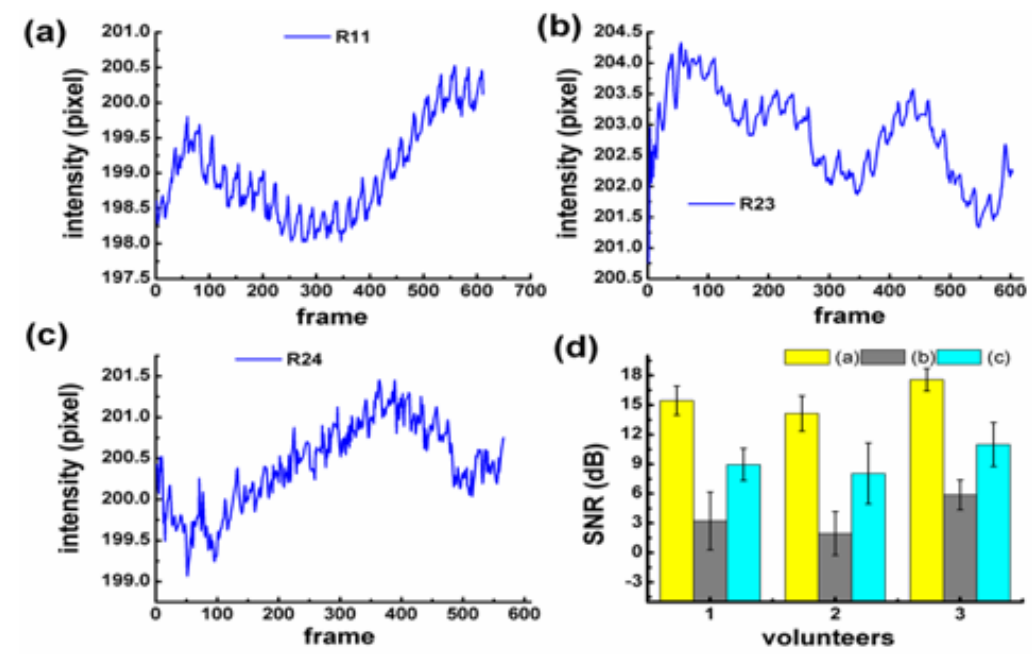

Figure 4: Results for the waveforms of finger(a), central palm(b) and palmar root(c), and their SNRs(d). In (a), (b) and (c), based on average pixel value of respective region, the waveforms are obtained. In (d), the value of SNR is averaged from ten measurements of three different volunteers, and their error bars also were showed.

\section{Self-Adaptive PPGi Denoise}

As shown in Figure 5a, the original waveform of PPGi signal from optimized ROI is obtained, in which the pulse wave is immersed in a large amount of noise. By comparing different levels and thresholds, the two-level Rig sure method of wavelet transform has better denoising results than others, and it is used to remove tiny glitches and make the waveform smoother, as shown in Figure 5b. Because the noises are in high-frequency, a Butterworth low-pass filter is constructed and used to filter out high-frequency noises, and at this point the waveform has become very smooth as shown in Figure $5 \mathrm{c}$. Besides, the median filter is needed to eliminate inclined phenomenon, and Figure $5 \mathrm{~d}$ shows the final result processed by the median filter.

\section{Blood Pressure Estimation}

A MATLAB program was developed to extract single cycle signals and calculate $\mathrm{Ts}$ and $\mathrm{Td}$, then calculate the corresponding I am using equation 3. Furthermore, according to the equations 2 and 3 and the data sets in the database, the values of $\mathrm{R}$ and $\mathrm{C}$ were obtained, and they are $\mathrm{R}=212.103$ ohms and $\mathrm{C}=0.847 \mathrm{~F}$, respectively. Finally, R, C, Ts, Td and I were inserted into equations 4,5 to estimate the high pressure (HP-PPGi) and low pressure (LPPPGi) values. To verify the suitability of the optimized ROI, the BP values of the forehead, finger, palm center, and palm root were calculated by the above-mentioned method, respectively. Further the calculated values of HP-PPGi and LP-PPGi are compared with the measured high pressure (HP-CSM) and low pressure (LP-CSM) 
which are measured by CSM. The correlations of the calculated data with the measured ones are examined by the error rates, as shown in Figssure 6. The error rate is defined as "|BP-CSM - BP-PPGi| / BP-CSM ", in which "BP" is HP and LP. It can be observed that lower error rates are from the ROIs of finger and palm root. According to the above results, it can be seen that the best error-rate comes from optimized RO can be controlled below $5 \%$.
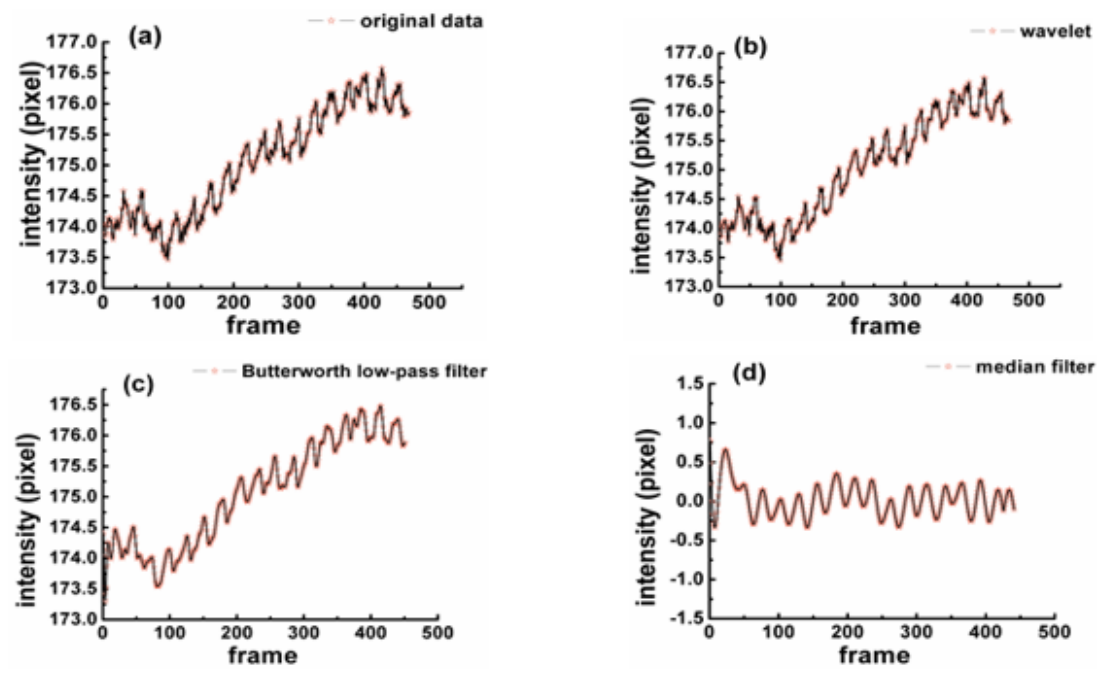

Figure 5: Results for the waveform of PPGi signal based on different denoising methods. In (a), the original waveform is obtained which derived from the video pixel mean. In (b), the wavelet transform is used to remove glitch noises. In (c), there is smooth waveform after high frequency noises are filtered by the Butterworth low-pass filter. In (d), in order to eliminate the baseline in the waveform, the median filter is applied.

\section{Discussion}

Since more distinct pulse wave with higher SNR can be collected from "hand" as shown in Figure 2, it is believed that "hand" is more suitable for PPGi analysis than "forehead". Further experiments found discrepancies are also existed when ROIs in the hand are changed, no matter what kind of illuminations are used, as shown in Figure 3. It is deduced to be caused by the different vascular density in different ROIs, because the different blood volume in them can change the PPGi amplitude, as illustrated in Figure 1b. Moreover, for the observed difference caused by varied light sources in Figures 3a-3d, it is interpreted according to the references $[28,29]$. Because the oxygenated hemoglobin and deoxyhemoglobin in blood have more higher absorbance for green light, in phase II their concentrations are decreased by the injected blood. This lowered concentration is closely related to the density of blood vessels. In the compact district, like R11, the hemoglobin is diluted more than in the sparse area like R23, then less absorbance of green light is in the compact district, therefore the collected reflected-green light is increased, and the waveform shows an upward trend. This deduction demonstrates R11 is a vascular dense area. More comparisons performed for R11, R21, R24, R34, etc. in Figure 4 demonstrate, there are more distinct pulse wave and higher SNR in R11, in consequence, R11 is determined as the optimized ROI [29,30].The effect of the self-adaptive denosing method is confirmed by Figure 5, based on the filtered PPGi signals from different ROIs (the forehead, finger R11, palm center R23, and palm root $\mathrm{R} 24$ ), the best calculated BP value are from R11, as shown in Figure 6, because the error rates of R11 are lower than other regions.
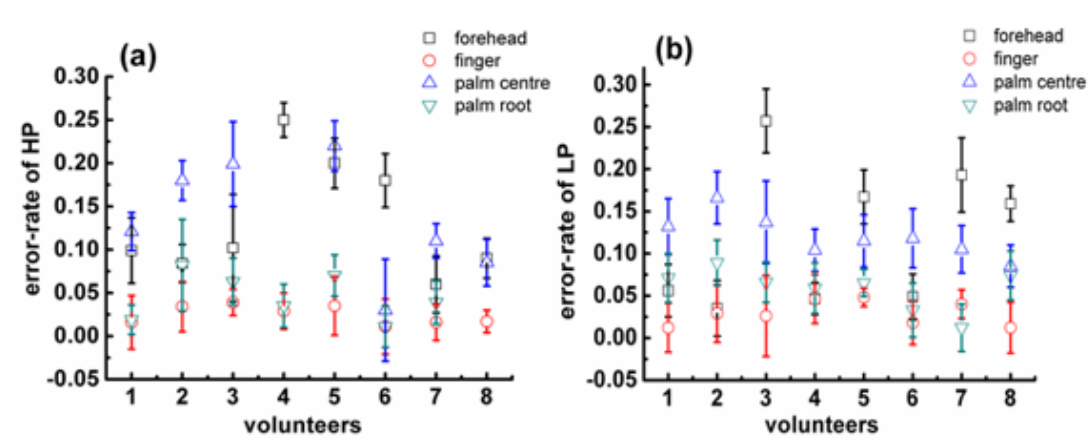

Figure 6: The deviation between calculated BP values for 8 volunteers and measured data are evaluated by using the error rates of HP (a) and LP (b). In (a) and (b), the calculated data are from different ROIs: forehead, finger, palm center and palm root. Error bars are the standard deviation for the average values $(n=10)$. 


\section{Conclusion}

The major improvement of this study is that it provided an optimization method for the ROI selection one the basis of factitious hemoperfusion for the purpose of increasing the PPGi signals' SNR and the accuracy of physiological parameters (blood pressure as a proof-of-concept), which are extracted from PPGi. The effectiveness of the proposed method was demonstrated by the agreements of the results from the repeated ROI optimizing experiments under four kinds of illuminations which are red, green, blue and white. Furthermore, a SNR self-adaptive PPGi denoising method was proposed and used to get ideal PPGi signal. Finally, the ROI optimized PPGi analysing method and self-adaptive PPGi denoising approach were integrated with Windkessel mathematical model to estimate BP. The error rate of the estimated BP from the optimized ROI is less than 5\%. Our next stage of this work is to test our ROI optimization method with more human subjects and also investigate its clinical performance. We believe that our method is beneficial to PPGi as well as its applications for vital signs' monitoring in clinical and domestic medicine.

\section{Acknowledgment}

This work is supported by the National Natural Science Foundation of China (Grant no.61371028, 61771260).

\section{References}

1. John (2007) Photoplethysmographic and its application in clinical physiological measurement. Physiol Meas 28(3): R1-39.

2. Yong Poh Yu, P Raveendran, Chern Loon Lim, Ban Hoe Kwan (2015) Dynamic heart rate estimation using principal component analysis. Biomed Opt Express 6(11): 4610-4618.

3. Matsumura, P Rolfe, S Toda, T Yamakoshi (2018) Cuffless blood pressure estimation using only a smartphone. Scientific reports 8(1): 7298.

4. Karlen A, Garde D, Myers C, Scheffer J, Ansermino M, et al. (2014) Respiratory rate assessment from photoplethysmographic imaging. in Proc $36^{\text {th }}$ Annual Int Conf of the IEEE Engineering in Medicine and Biology Society Germany 2014:5397-5400

5. Alian K, Shelley H (2014) Photoplethysmographic. Best Pract Res Clin Anaesthesiol 28(4): 395-406.

6. Liu H, Wang Y, L Wang (2012) A review of non-contact, lowcost physiological information measurement based on photoplethysmographic imaging. in Proc Annual Int Conf of the IEEE Engineering in Medicine and Biology Society Germany 2012: 2088-2091.

7. Daniel McDuff, Gontarek S, Picard RW (2014) Improvements in remote cardiopulmonary measurement using a five band digital camera. IEEE Trans Biomed Eng 61(10): 593-2601.

8. Kenta Matsumura, Peter Rolfe, Jihyoung Lee, Takehiro Yamakoshi (2014) iphone 4s photoplethysmographic: which light color yields the most accurate heart rate and normalized pulse volume using the iphysiometer application in the presence of motion artifact? PloS one 9(3): e91205.

9. McDuff D, Gontarek S, Picard RW (2014) Remote detection of photoplethysmographic systolic and diastolic peaks using a digital camera. IEEE Trans Bio Med Electron 61(12): 2948-2954.

10. Nam Y, Nam YC (2017) Photoplethysmographic signal analysis for optimal region-of-interest determination in video imaging on a built-in smartphone under different conditions. Sensors (Basel, Switzerland) 17(10): 2385-2403.

11. Verkruysse W, Svaasand LO, Nelson JS (2008) Remote plethysmography imaging using ambient light. Opt Express 16(26): 21434-21445.
12. Cennini G, Arguel J, Aksit K, Van Leest A (2010) Heart rate monitoring via remote photoplethysmographic with motion artifacts reduction. Opt Express 18(5): 4867-4875.

13. Audrey Chung, Xiao Yu Wang, Robert Amelard, Christian Scharfenberger, Joanne Leong et al. (2015) High-resolution motion-compensated imaging photoplethysmographic for remote heart rate monitoring. International Society for Optics and Photonics 9316.

14. Kamshilin A, Miridonov S, Teplov V, Saarenheimo R, and Ervin Nippolainen (2011) Nippolainen Photoplethysmographic imaging of high spatial resolution. Biomed Opt Express 2(4): 996-1006.

15. Frédéric Bousefsaf, Choubeila Maaoui, Alain Pruski (2013) Continuous wavelet filtering on webcam photoplethysmographic signals to remotely assess the instantaneous heart rate. Biomedical Signal Processing and Control 8(6): 568-574.

16. Lee CM, Zhang YT (2003) Reduction of motion artifacts from photoplethysmographic recordings using a wavelet denoising approach. IEEE EMBS Asian Pacific Conference on Biomedical Engineering Kyoto Japan.

17. Ryan Imms, Sijung Hu, Vicente Azorin Peris, Michaël Trico, Ron Summers (2014) A high performance biometric signal and image processing method to reveal blood perfusion towards $3 \mathrm{~d}$ oxygen saturation mapping. The International Society for Optical Engineering pp. 8947.

18. Shao D, Yang Y, Liu C, Tsow F, Yu H, et al. (2014) Noncontact monitoring breathing pattern, exhalation flow rate and pulse transit time. IEEE Trans Bio Med Electron 61(11): 2760-2767.

19. McDuff Daniel J, Blackford EB, Estepp JR (2018) Fusing partial camera signals for noncontact pulse rate variability measurement. IEEE Transactions on Biomedical Engineering 65(8): 1725-1739.

20. McDuff J, Estepp JR, Piasecki AM, Blackford EB (2015) A survey of remote optical photoplethysmographic imaging methods. Proc $37^{\text {th }}$ Annual Int Conf of the IEEE Engineering in Medicine and Biology Society (EMBC) Milan Italy.

21. Lewis T (1926) The blood vessels of the human skin. Br Med J 2(3418): 61-62.

22. Siegfried Kästle, Friedemann Noller, Siegfried Falk, Anton Bukta, Eberhard Mayer, Dietmar Miller (1997) A new family of sensors for pulse oximetry. Hewlett-Packard Journal 48(1): 39.

23. Yansun Xu,Weaver JB, Healy DM, Jian Lu (1994) Wavelet transform domain filters: A spatially selective noise filtration technique. Image Processing, IEEE Transactions on 3(6): 747-758.

24. Selesnick IW, Burrus CS (1996) Generalized digital butterworth filter design. IEEE Transactions on Signal Processing 46(6): 1367-1370.

25. Lee CN, Jen W (1992) Bit-sliced median filter design based on majority gate. IEEE proceedings-G. 139: 63-71.

26. Westerhof N, Lankhaar JW, Westerhof BE (2009) The arterial windkessel, Med. Biol. Eng. Comput. 47(2): 131-141.

27. Choudhury AD, Banerjee R, Sinha A, Kundu S(2014) Estimating blood pressure using Windkessel model on photoplethysmogram. Annual International Conference of the IEEE Engineering in Medicine and Biology Society, Germany 2014: 4567-4570.

28. Liu D, Gorges MG, Jenkins SA (2012) University of queensland vital signs dataset: Development of an accessible repository of anesthesia patient monitoring data for research. Anesth Analg 114(3): 584-589.

29. Faber DJ, Mik EG, Aalders MC, van Leeuwen TG (2003) Light absorption of (oxy-)hemoglobin assessed by spectroscopic optical coherence tomography. Opt Lett 28(16): 1436-1438.

30. Little MA, Sprangel C (1980) Skin reflectance relationships with temperature and skinfolds. Am J Phys Anthropol 52(1): 145-151. 
ISSN: 2574-1241

DOI: 10.26717/BJSTR.2019.13.002356

Yunfang Jia, Xiaoshan Zhu. Biomed J Sci \& Tech Res

(C) (i) This work is licensed under Creative

Submission Link: https://biomedres.us/submit-manuscript.php

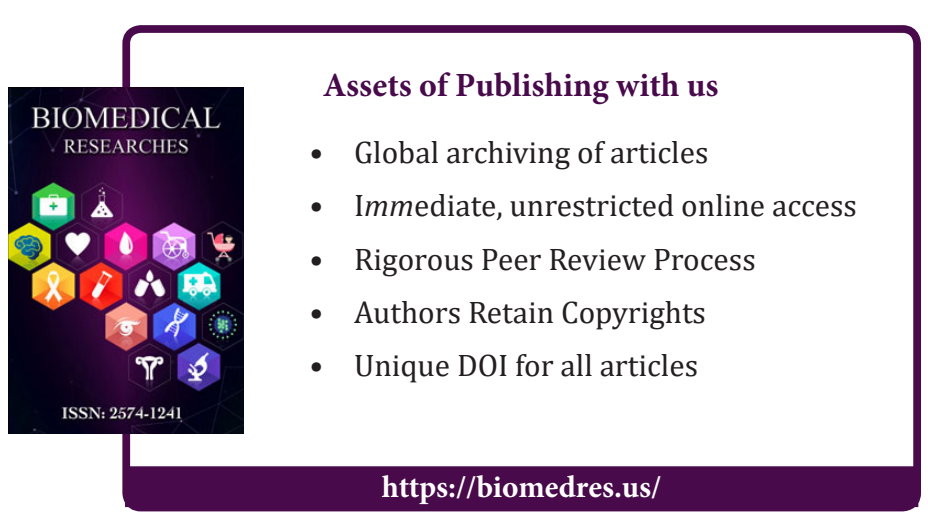

